\title{
Evaluación de la Climatización en Locales Comerciales, Integrando Técnicas de Termografía, Simulación y Modelado por Elementos Finitos
}

\author{
José A. Madrigal(1), Juan J. Cabello(2)*, Alexis Sagastume ${ }^{(2)}$ y Milen Balbis ${ }^{(2)}$. \\ (1) Univ. Nacional Autónoma de México (UNAM), Fac. de Ingeniería, Dpto. Proceso y Uso Eficiente de \\ Energía. Av. Universidad 3000, Ciudad Universitaria, Coyoacán. México. \\ (e-mail: jamadrigal1988@gmail.com) \\ (2) Univ. de la Costa, Fac. de Ingeniería, Dpto. de Energía. Calle 58 No. 55-66, Barranquilla. Colombia. \\ (e-mail: jcabello2@cuc.edu.co; asagastume1@cuc.edu.co; mbalbis1@cuc.edu.co)
}

* Autor a quien debe ser enviada la correspondencia

Recibido Nov. 9, 2017; Aceptado Ene. 17, 2018; Versión final Mar. 15, 2018, Publicado Ago. 2018

\begin{abstract}
Resumen
En este estudio que se presenta se integran técnicas utilizadas para analizar problemas asociados a la climatización que por sí no son suficientes para evaluar todo un sistema de acondicionamiento de aire. Se utiliza la termografía para obtener el perfil de temperatura en el área de incidencia del aire climatizado y los focos calientes en el interior del local. Mediante la simulación con el software Trnsys se obtiene el comportamiento de la temperatura interior y se estima el perfil de carga térmica. Con el diseño asistido por computador se crea un modelo espacial de la zona térmica y con el método de los elementos finitos se modela la circulación del aire y la distribución de la temperatura en el espacio. Finalmente, se propone un nuevo esquema para el sistema de frío a partir de los resultados obtenidos con las herramientas anteriores.
\end{abstract}

\section{Evaluation of Air Conditioning in Commercial Buildings, Integrating Thermography Techniques, Simulation and Modeling by Finite Elements}

\begin{abstract}
This study integrates different techniques to analyze some issues of the air conditioning system of a commercial establishment. A thermographic camera is used to assess the temperature profile in the area covered by the air conditioning system and also to detect the higher temperature spots. The air conditioning system of the facility is simulated using the software Trnsys to estimate the indoor temperature and the thermal load profile. Afterward, using computer-aided design, a model of the air circulation in the thermal zone is developed, which is simulated using the finite element method to obtain the temperature profile in the area. As a result, a new operational scheme of the air conditioning that improves the temperature profile is proposed.
\end{abstract}

Keywords: thermography; simulation; air-conditioning; thermal load; temperature profile 


\section{INTRODUCCIÓN}

Los locales comerciales son los encargados de suplir, a través de su actividad económica, las necesidades de productos y servicios básicos de la población. Para alcanzar el bienestar térmico y la satisfacción de los clientes, así como la conservación de los productos comerciales, es necesario acondicionar el ambiente en el interior del local comercial. El local comercial estudiado se encuentra ubicado en la provincia de Cienfuegos, donde la temperatura media anual es de $25^{\circ} \mathrm{C}$ y en verano los extremos de calor alcanzan los $35^{\circ} \mathrm{C}$ con una humedad relativa del $80 \%$. Las características mencionadas hacen de Cuba un país tropical donde el enfriamiento con deshumidificación, como proceso para acondicionar el aire, en el interior de locales se presente como una necesidad siempre que se quiera cumplir con el criterio de confort térmico (Carbonell y Salgado, 2016). En los locales comerciales de pequeña dimensión se utilizan fundamentalmente los minisplits para cumplir con estos criterios. Los estándares presentados por la Sociedad Americana de Ingenieros de Calefacción, Refrigeración y Aire Acondicionado (ASHRAE por sus siglas en ingles), y otras investigaciones describen el confort térmico cualitativamente como la "condición de la mente que expresa satisfacción con el ambiente térmico". (ASHRAE, 2005; ASHRAE, 1997; Bush, 1992), (Djamila, 2017; Van Hoof, et al., 2017; Viloria et al., 2016; Cabello et al., 2016). Establecer un valor de temperatura para garantizar el confort óptimo en locales comerciales se presenta complejo, debido a que este se puede desplazar, por su dependencia con el microclima del lugar, usos y costumbres, estilo de vida, edad o constitución física de las personas. Por tanto, asumir el valor recomendado según los estándares internacionales, aunque se presenta como una vía fácil de aplicar, no se considera recomendable para generalizar (Ramos, 2012), y en el caso de nuestro estudio por ser realizado en la ciudad de Cienfuegos, se utilizará $24^{\circ} \mathrm{C}$ como valor de temperatura límite para el confort térmico.

Durante la etapa de diseño del sistema de climatización se tienen en cuenta las características constructivas del local, el servicio que se va a prestar, la ocupación, la ubicación geográfica y otras importantes consideraciones (ASHRAE, 1997; Yin et al., 2016; Vakiloroaya et al., 2014; Barros et al., 2017; Shan y Shengwei, 2017); sin embargo, para los locales comerciales de pequeñas dimensiones el criterio económico es un factor determinante en la selección del sistema de climatización. En Cuba, las empresas encargadas de la instalación de los sistemas de climatización solo determinan la carga térmica puntual en un día cualquiera del año y seleccionan un esquema de clima que supere dicho valor, desatendiendo así la variabilidad que tiene el clima en las diferentes épocas del año. En cualquier aplicación los sistemas de acondicionamiento de aire representan un alto costo dentro de la estructura de gastos (Fernández et al., 2014; Cabrera et al., 2004; Allouhi, 2015), por lo cual se requiere de técnicas que permitan evaluar cuantitativamente y cualitativamente los sistemas que están en funcionamiento. Cualquier mejora en el sistema de climatización puede ser traducida en ahorros monetarios para la empresa y en mejoras de confort para los usuarios.

En el presente trabajo, a través del uso integrado de la simulación de cargas térmicas mediante el software Trnsys (Klein et al., 2018), la termografía como técnica para observar la incidencia del aire frío y los puntos calientes, y el método de los elementos finitos para evaluar la distribución volumétrica del aire frío y la circulación del mismo, se identifican las ineficiencias existentes debido al diseño del sistema de aire acondicionado en un local comercial y se proponen mejoras que permiten garantizar el confort.

\section{MATERIALES Y MÉTODOS}

La simulación de cargas térmicas mediante el uso del software Trnsys, por la flexibilidad que presenta para simular el comportamiento de sistemas transitorios y por contar con una amplia librería de componentes energéticos, frente a otros software de simulación que se centran en la evaluación del rendimiento de los sistemas de energía térmica y eléctrica, es uno de los recursos disponibles más expandidos y utilizados por diferentes autores para la determinación de cargas térmicas (Oldewurtel et al., 2010; Privara et al., 2013; Vehelts, 2012; Vega et al., 2015; Gallegos et al., 2010). El objetivo fundamental de la simulación térmica en una instalación, es obtener un modelo dinámico de algunas variables del sistema (carga térmica, temperatura interior, humedad relativa, coeficientes de transferencia de calor, entre otras). Según (Vega et al., 2015), los procesos de transferencia de calor pueden ser analizados como problemas transitorios, o sea, fenómenos que cambian constantemente en el tiempo independientemente de su mecanismo y su magnitud.

Con la simulación a través del software Trnsys se puede obtener un perfil de temperatura variable en el tiempo, sin embargo, es imposible obtener un perfil de distribución volumétrica de la temperatura operativa en el interior y las zonas muertas, lugares donde puede verse afectado el confort térmico de las personas. La inspección térmica de una instalación, basada en el uso de la termografía, ofrece la posibilidad de realizar un análisis no invasivo donde se pueden obtener variables determinantes en la carga térmica del local como: focos calientes puntuales, distribución de temperaturas superficiales, infiltraciones de calor e incidencia de las venas de aire frío proveniente del sistema de acondicionamiento del aire (Sales, 2017; Maroy, 2017). En la elaboración del presente estudio se utilizó una cámara infrarroja. La Tabla 1 muestra las especificaciones técnicas del equipo utilizado para la medición. 
Tabla 1: Datos técnicos de la cámara infrarroja.

\begin{tabular}{|l|l|}
\hline \multicolumn{1}{|c|}{ Características } & \multicolumn{1}{c|}{ Valores } \\
\hline Sensibilidad térmica & $<80 \mathrm{mk}$ a $30^{\circ} \mathrm{C}$ \\
\hline Tipo de detector & FPA $160 \times 120$ píxeles, a-Si \\
\hline Rango temperatura & $\mathrm{De}-20$ a $100^{\circ} \mathrm{C} / \mathrm{de} 0$ a $280^{\circ} \mathrm{C}$ \\
\hline Exactitud & $\pm 2{ }^{\circ} \mathrm{C}$ \\
\hline
\end{tabular}

A pesar de las potencialidades ofrecidas por la cámara, la termografía por sí sola no es una herramienta que permita caracterizar un sistema de climatización, por lo que es necesario el uso de otras herramientas que analicen la información recogida en las imágenes térmicas. A través del diseño asistido por ordenador (CAD por sus siglas en inglés) se crea un modelo tridimensional de la zona térmica. La Fig. 1, representa el esquema de análisis, donde se introducen los valores reales de velocidad y temperatura medidos in situ y los parámetros de clima del sistema obtenidos con las herramientas antes mencionadas.

EI MEF es la herramienta usada para solucionar los problemas asociados a la mecánica de los fluidos computacional (CFD por sus siglas en inglés), siendo capaz de realizar análisis en régimen estacionario y transitorio (Wu et al., 2017).

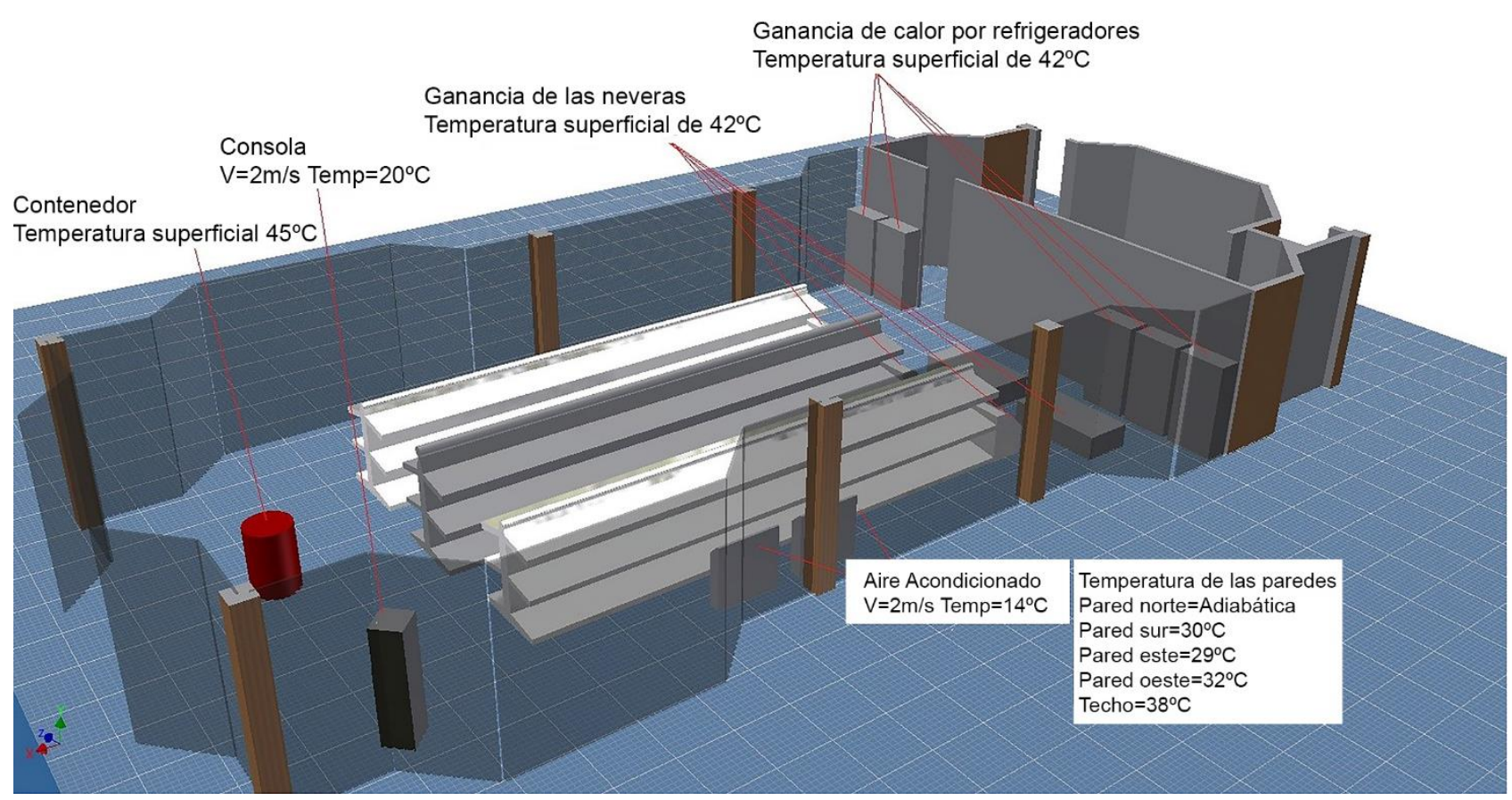

Fig. 1: Modelo tridimensional del mercado.

El local comercial estudiado presenta como rasgo distintivo, la gran área acristalada que posee, llegando a representar alrededor del $90 \%$ de su superficie lateral total. Como consecuencia de la arquitectura del local, es necesario un uso intensivo de la climatización lo que genera un elevado consumo de energía eléctrica para satisfacer este servicio. El mismo alcanza hasta el $62 \%$ del consumo total de la entidad. Se encuentra ubicado hacia el norte cardinal, con la puerta de acceso hacia el sur. Las paredes este y oeste son las de mayor área y el cristal en las mismas representa el $94 \%$ del área total. El recinto cuenta con un volumen de $550 \mathrm{~m}^{3}$ y un régimen de trabajo diario de 10 horas.

Para la modelación se tomaron 10 renovaciones del aire por hora según las recomendaciones de la ASHRAE y un cristal doble transparente con un espesor de $8 \mathrm{~mm}$ igual al que se encuentra en el local comercial. La humedad relativa promedio de la zona donde se encuentra enclavado el local comercial es de $82 \%$ como promedio anual, la temperatura promedio es de $32{ }^{\circ} \mathrm{C}$ en verano y $27^{\circ} \mathrm{C}$ en invierno. La Fig. 2 muestra la distribución de la radiación solar por las diferentes paredes en el mes de julio, siendo la pared oeste la que más radiación recibe. 


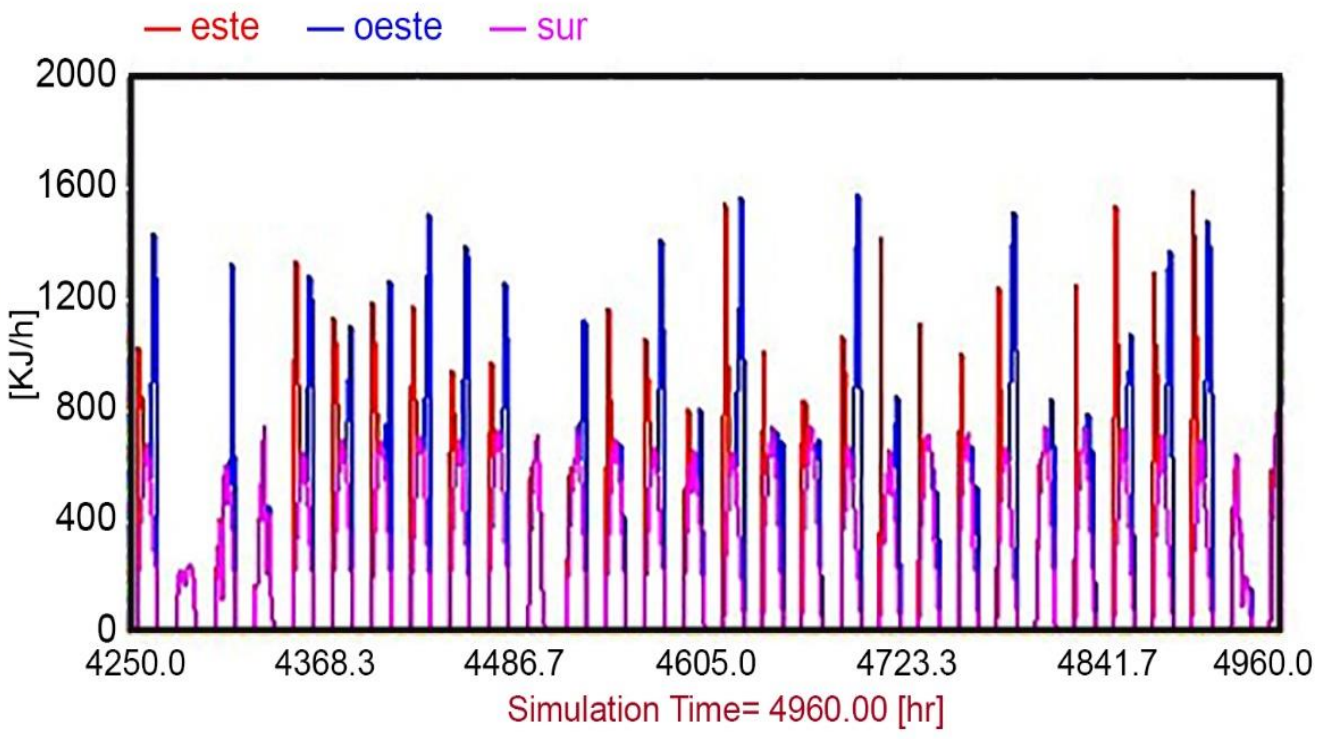

Fig. 2: Distribución radiación solar por las paredes del local.

\section{RESULTADOS Y DISCUSIÓN}

El local cuenta con una capacidad instalada de $35 \mathrm{~kW}$ de refrigeración, suministradas por 2 minisplits de 5 toneladas de refrigeración cada uno. Para obtener un modelo dinámico del comportamiento anual de la temperatura en el interior del local, se utilizó el software Trnsys. El mismo utiliza variables determinantes en el comportamiento de los sistemas de climatización: esquema constructivo del local, nivel de infiltraciones, ganancias de calor, materiales de las paredes y ventanas, ventilación y variables meteorológicas de la provincia en un año típico.

Al realizar una simulación inicial se puede observar que el pasillo 1 y 2 son las zonas más críticas del local comercial y no se garantiza la temperatura de confort adecuada. La Fig. 3 muestra que el mes de julio se reportan los valores más elevados los cuales sobrepasan los $33^{\circ} \mathrm{C}$.

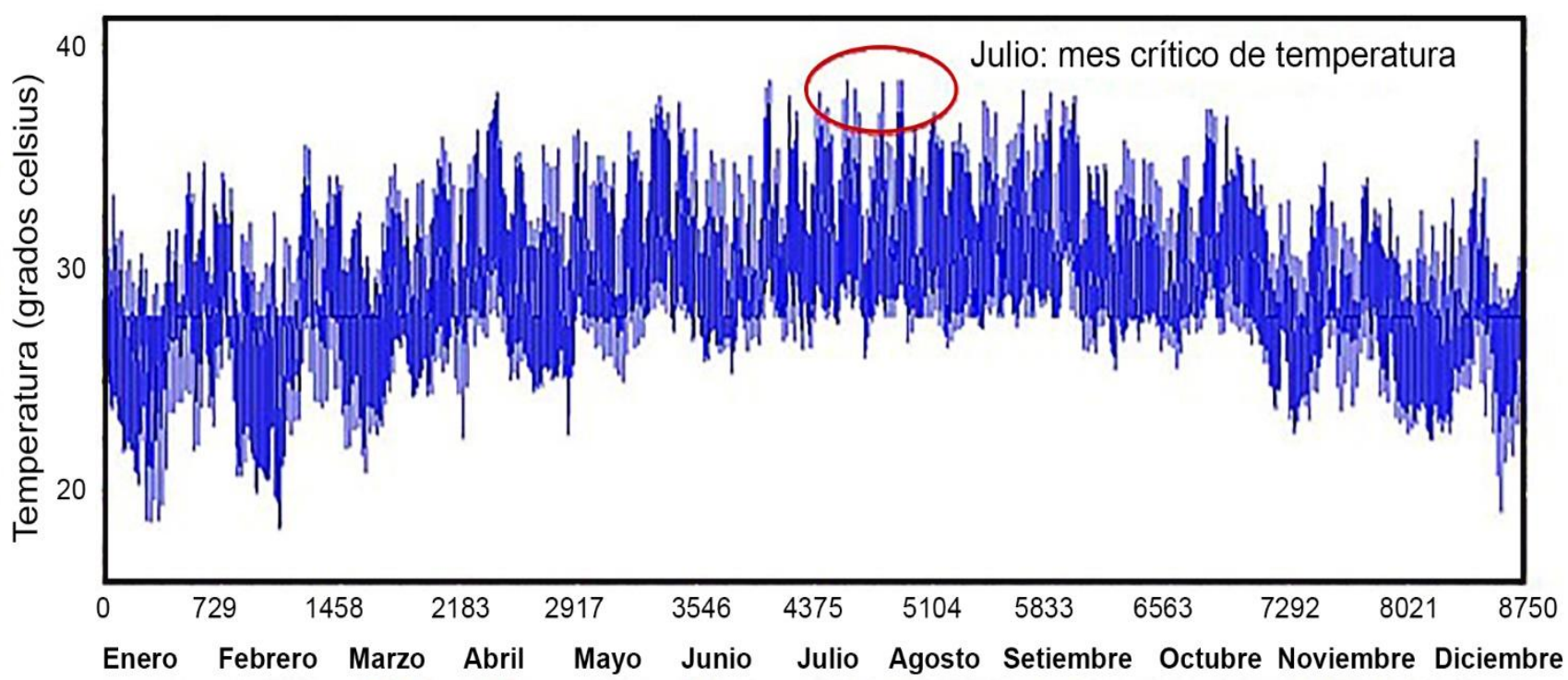

Fig. 3: Comportamiento anual de la temperatura en el interior del local.

La Fig. 4 muestra el comportamiento de la temperatura en esa zona del local durante las 24 horas del día crítico del año, correspondiente al 23 de Julio, y el proceso de acumulación de calor que se produce hasta alcanzar un valor tope de $36{ }^{\circ} \mathrm{C}$, con un promedio en la jornada laboral de $34{ }^{\circ} \mathrm{C}$. 


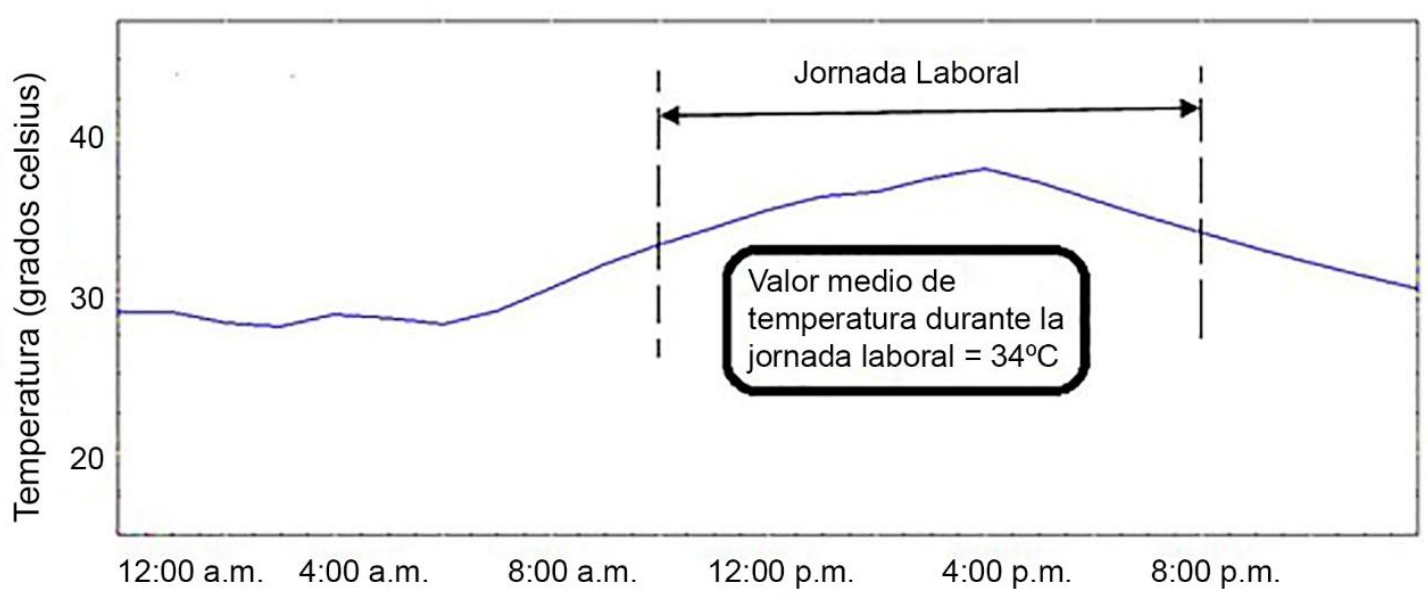

Fig. 4: Comportamiento de la temperatura en el interior del local el día crítico.

Durante el estudio termográfico realizado se evidenció la no existencia de homogeneidad en la distribución del aire climatizado ocasionando notables diferencias en la distribución superficial de temperatura. La Fig. 5 muestra una comparación de los resultados obtenidos con la termografía y la modelación de la distribución de temperatura con el método de los elementos finitos (MEF), puede observarse que en el pasillo 1, los minisplits impulsan directamente el aire frío hacia el estante, provocando como resultado una obstaculización del fluido e impidiendo la correcta circulación en el interior del local. Tal situación provoca que la distribución de temperatura en el pasillo 2, no se comporte de manera homogénea, con zonas donde la temperatura está alrededor de los $22^{\circ} \mathrm{C}$ y otras donde los valores de temperatura están alrededor y sobre los $30^{\circ} \mathrm{C}$.
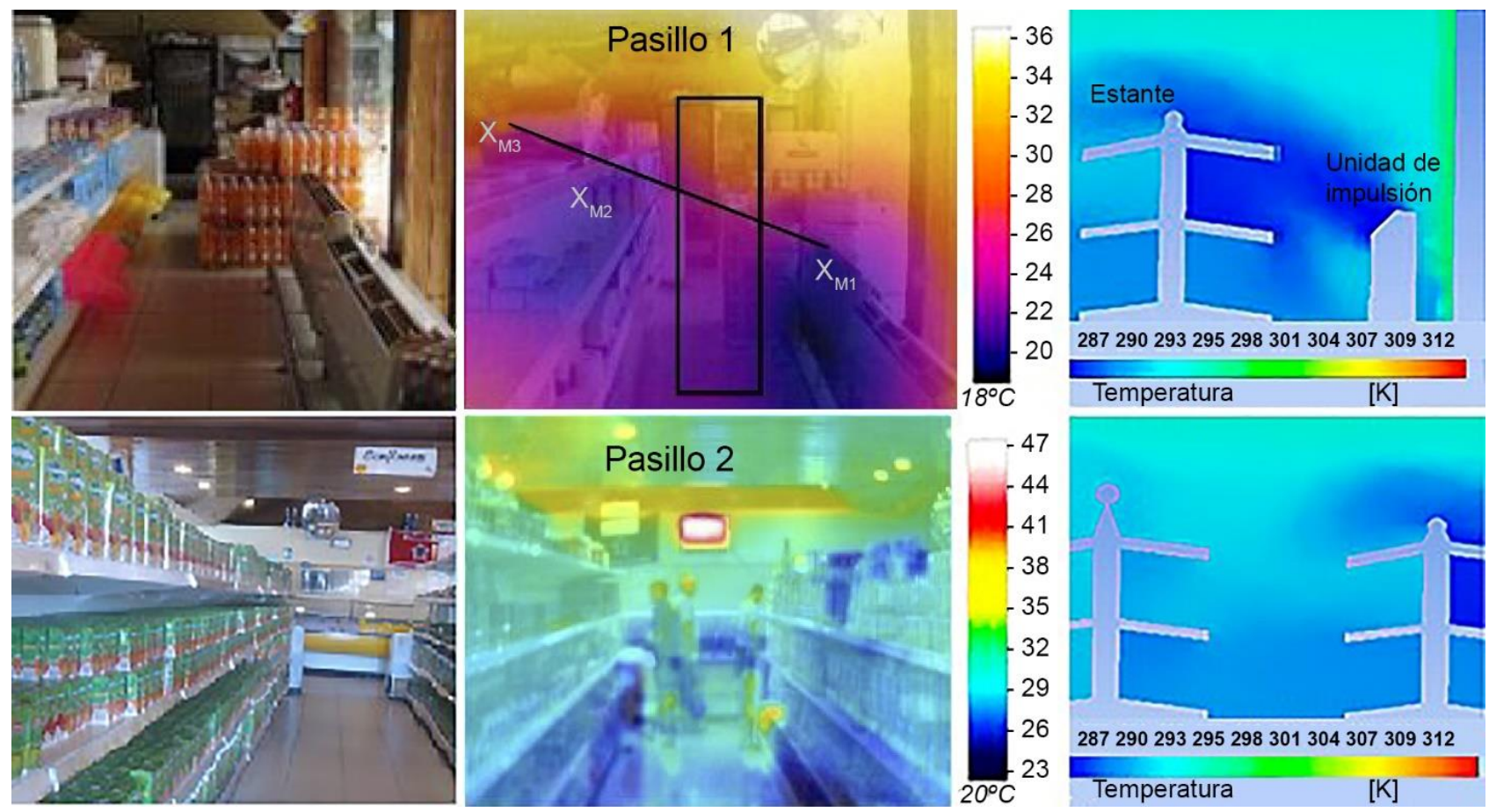

Fig. 5: Imágenes reales, termográfica y con el método de los elementos finitos de los pasillos 1 y 2.

La simulación de la circulación de aire y la temperatura volumétrica se obtienen a partir de un análisis estacionario para determinar la circulación de las venas de frio al interior del local, utilizando como fronteras las paredes exteriores del local donde no existen grandes sombras y las variables obtenidas con las herramientas anteriores: temperatura de salida del aire frío, velocidad del fluido y fuentes internas de calor. La Fig. 6 muestra que la circulación del aire no es adecuada, debido a que los estantes obstaculizan el movimiento de las venas de aire frío, y no permiten que estas se mezclen lo suficiente con el aire caliente dentro del local.

Al validar la no existencia de condiciones térmicas de confort en el interior del local debido a la incorrecta ubicación de las unidades terminales de impulsión (UTIs) y una insuficiente carga de refrigeración instalada, se determina la potencia de climatización necesaria para satisfacer las necesidades de frío y se propone un nuevo esquema para el sistema de distribución del aire climatizado. 


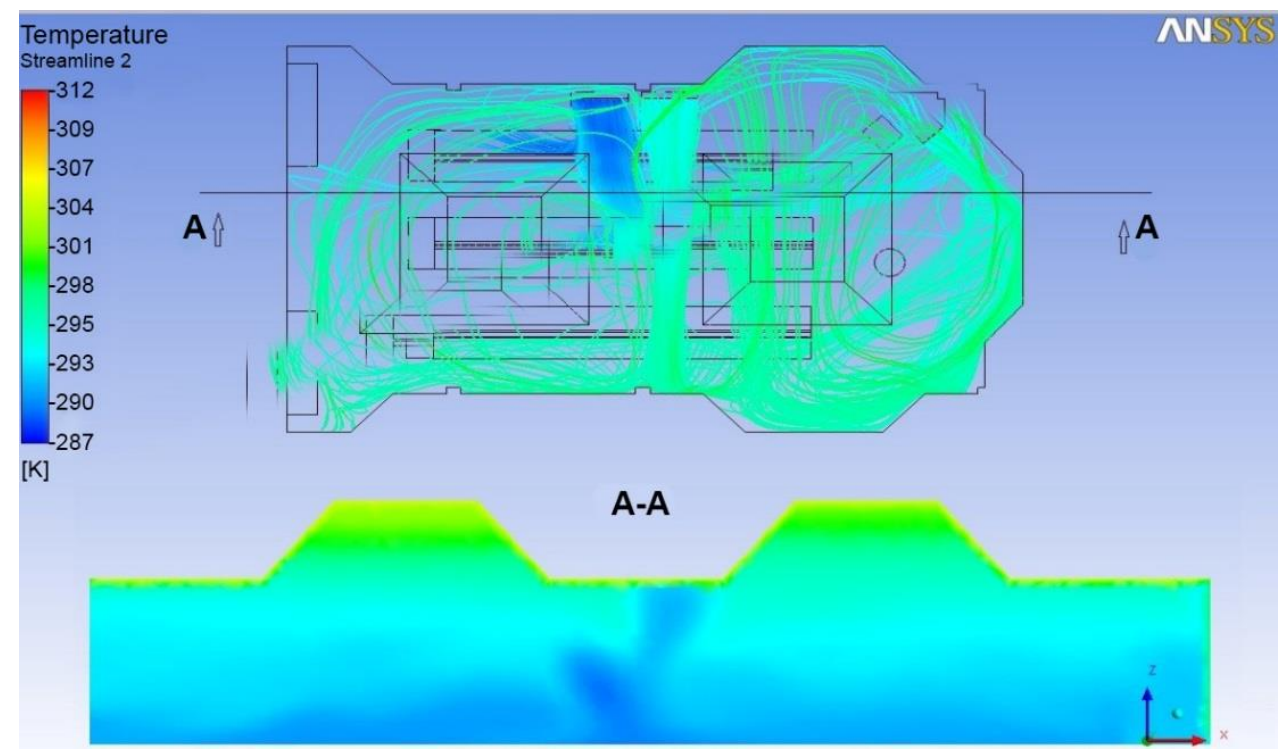

Fig. 6: Resultados de la simulación con el MEF.

Para determinar la potencia de frío a instalar mediante Trnsys, se fijó un valor esperado de $24^{\circ} \mathrm{C}$ en el interior del local y se simuló el comportamiento de la carga térmica durante la etapa comprendida entre los últimos días de junio y el mes de julio, ya que anteriormente durante este tiempo se habían reportado los mayores valores de temperatura. Un sistema de climatización que supere la demanda térmica para el período anterior garantiza valores de temperatura dentro del rango de confort, aunque introduce un cierto grado de sobredimensionamiento durante los meses de invierno.

Otras variables que se tuvieron en cuenta para la simulación con el software Trnsys fueron: presencia de 20 personas en el interior del local realizando la actividad comercial, existencia de 10 fuentes de calor internas con régimen de trabajo continuo (correspondientes a los compresores de las máquinas de refrigeración) y la ubicación de una cortina de viento en el acceso principal de manera tal que se disminuyera el valor de las infiltraciones de calor. La Fig. 7 muestra que para obtener $24^{\circ} \mathrm{C}$ en el interior del local según los resultados del software Trnsys y con las condiciones descritas anteriormente se necesita vencer una carga térmica máxima de $197000 \mathrm{~kJ} / \mathrm{h}$, lo cual equivale a un sistema de clima con una potencia instalada de 16 toneladas de refrigeración $(56.2 \mathrm{~kW})$.

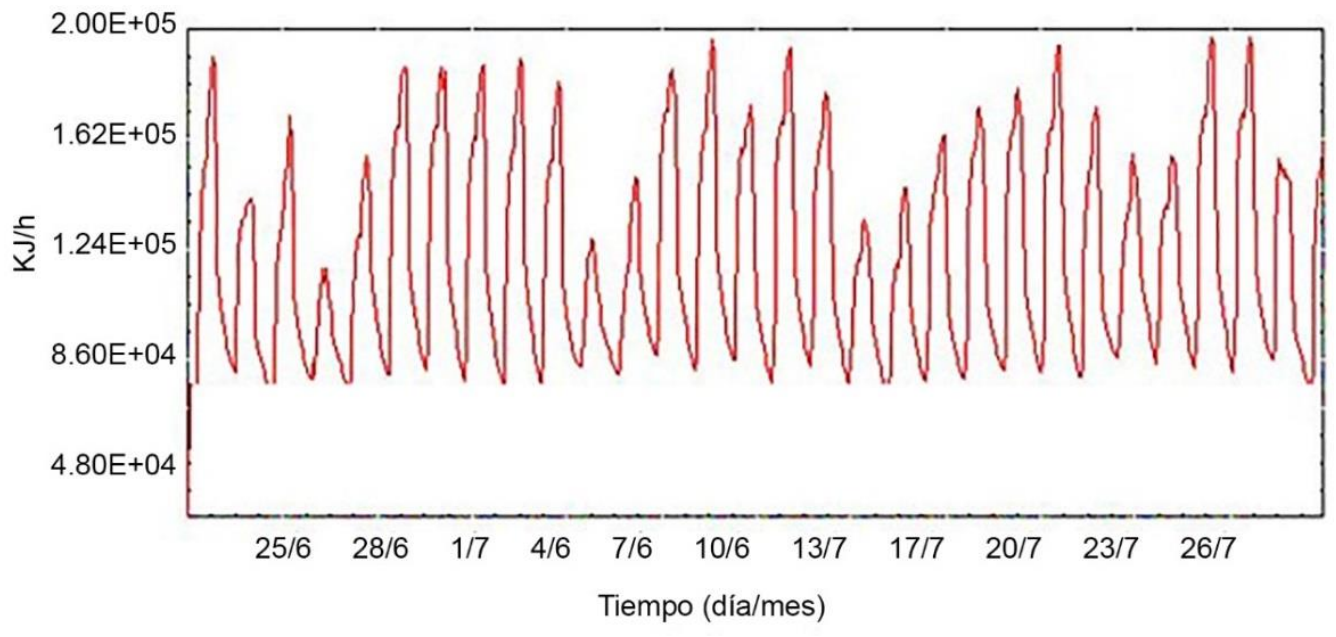

Fig. 7: Simulación de la carga térmica en el interior del local.

La recomendación realizada a los responsables por el local comercial fue la instalación de 4 minisplits de 5 toneladas de refrigeración cada uno, para una capacidad total de 20 toneladas de refrigeración $(70.3 \mathrm{~kW})$ garantizando vencer la carga térmica. Aunque el criterio utilizado introduce un elevado grado de sobredimensionamiento en los meses de invierno, esta decisión respalda un adecuado régimen de trabajo alterno de los equipos que permita desarrollar el plan de mantenimiento sin tener que afectar el confort térmico en la instalación. Una prueba realizada antes de proponer un nuevo esquema para el sistema de clima fue observar que pasaría con la circulación del aire y la distribución de temperatura en el pasillo principal si los equipos se mantuvieran en la posición actual, aunque con una mayor potencia de frío. 
La Fig. 8 muestra que los resultados obtenidos son mejores que la situación que actualmente presenta el local comercial, aunque se observa una distribución no homogénea de la temperatura a lo largo de todo el pasillo, ya que, existen $5^{\circ} \mathrm{C}$ de diferencia entre la zona de circulación del aire climatizado y otros puntos ubicados en el mismo pasillo. Tales resultados indican la necesidad de una nueva ubicación para las unidades terminales de impulsión (UTIs).

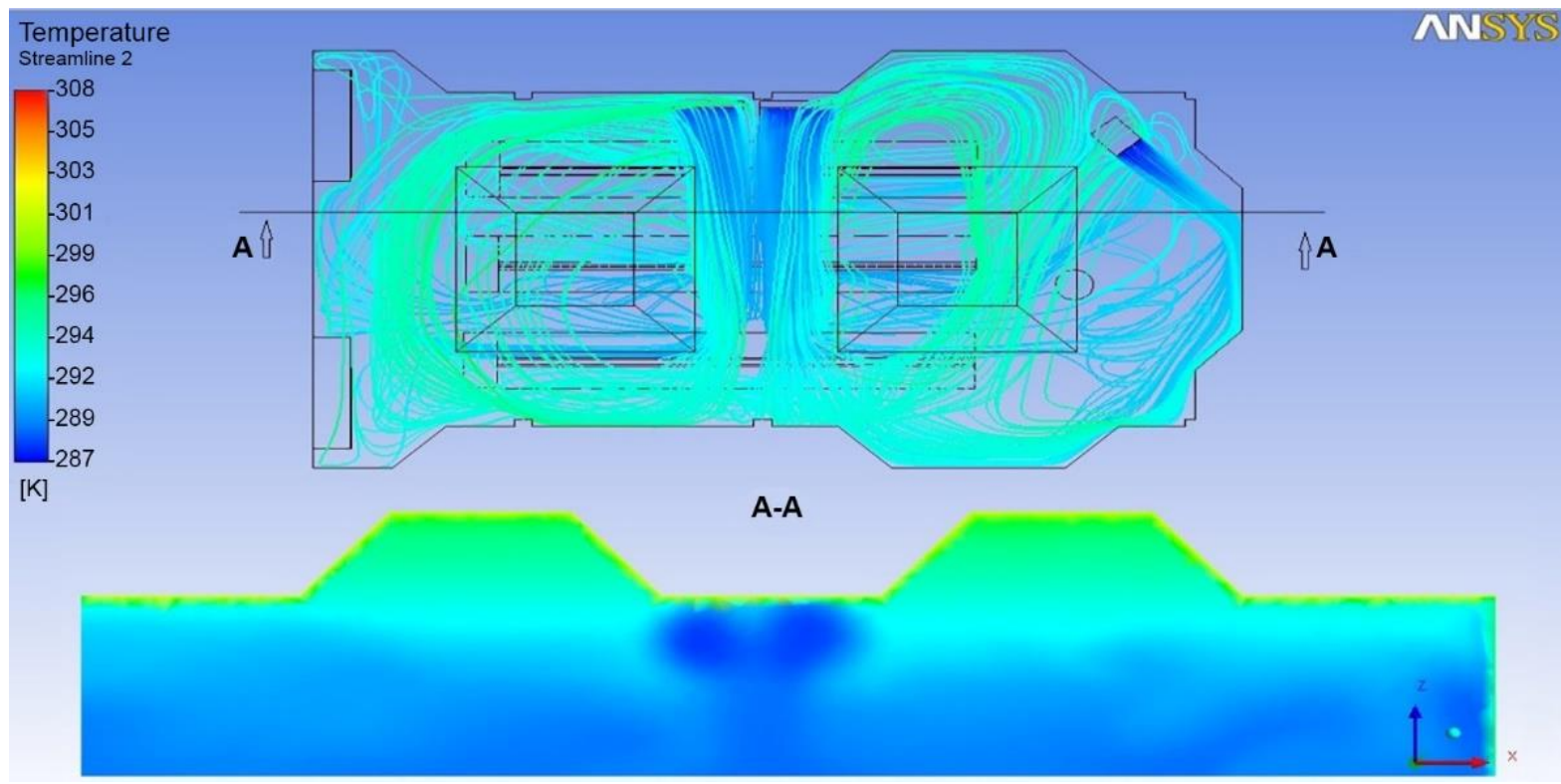

Fig. 8: Circulación del aire y distribución de temperatura en el pasillo.

Dado que el tipo de vena de aire que impulsan estos equipos es horizontal y no isoterma, se decidió que la disposición de los equipos fuera transversal a los obstáculos con la circulación del aire paralela a los estantes, De esta manera, la vena de aire frío impulsada no es obstaculizada y puede adentrarse lo suficiente en el local para que ocurra una buena mezcla. La pared interior propuesta para ubicar los equipos es la norte, a 0.2 metros del techo y con el fluido circulando directamente hacia los pasillos. De esta manera se garantiza un mayor alcance del aire frío, mejor mezcla y se eliminan las zonas muertas en la circulación. La Fig. 9 muestra la circulación del aire frío en el interior del local y la distribución de temperatura espacial del pasillo principal según los resultados obtenidos con el MEF.

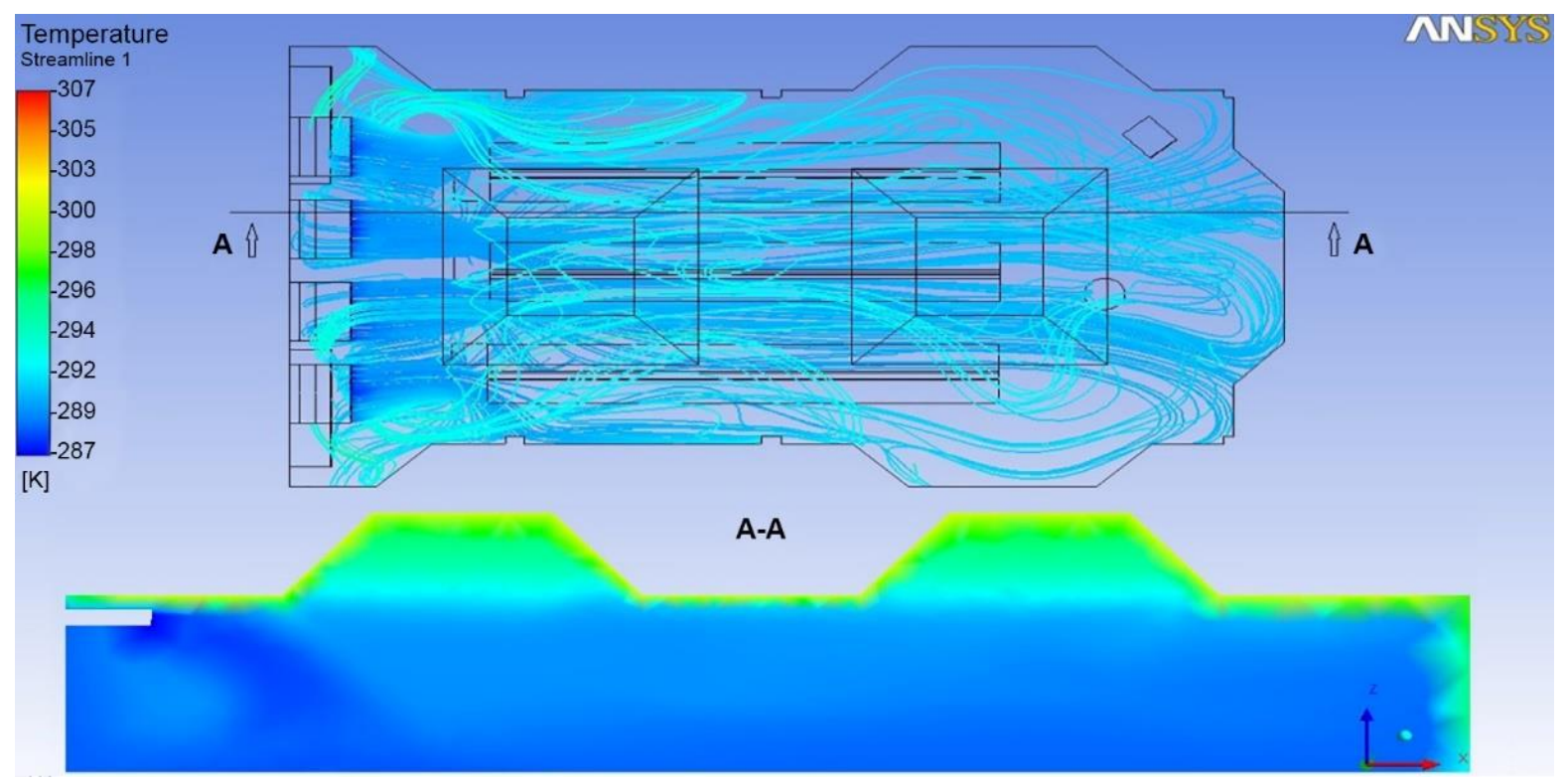

Fig. 9: Nuevo esquema del sistema de climatización y distribución de temperatura en el pasillo.

El nuevo esquema muestra un comportamiento homogéneo de la temperatura a lo largo del plano vertical del pasillo principal, sin grandes diferencias térmicas y con valores de temperaturas dentro de los parámetros de confort esperados. 


\section{CONCLUSIONES}

No es posible utilizar la técnica de termografía, la simulación con el software Trnsys o la modelación con el método de elementos finitos (MEF) como herramientas individuales para caracterizar de forma adecuada un sistema de climatización. Para la simulación de cargas térmicas se necesitan los puntos calientes y las infiltraciones obtenidas con la termografía, así como para la modelación del aire en el interior del local con el MEF se necesita la termografía para validar los resultados. En el caso de estudio es insuficiente la capacidad de frío instalada para satisfacer las necesidades de confort en el interior del local en las condiciones actuales, lo cual queda evidenciado con las altas temperaturas operativas obtenidas a partir de la simulación realizada con el software Trnsys y las mediciones realizadas. Los resultados obtenidos con las imágenes termográficas y la modelación con el MEF demostraron que es inadecuada la ubicación de las unidades terminales de impulsión. Para satisfacer las condiciones térmicas de confort en el caso de estudio es necesario instalar 20 toneladas de refrigeración $(70.3 \mathrm{~kW})$ distribuidas en cuatro equipos de 5 toneladas de refrigeración cada uno, los que deben ser ubicados a 0.2 metros del techo y dirigiendo la vena de aire de forma paralela a los pasillos.

\section{REFERENCIAS}

Allouhi, A., T. Kousksou, A. Jamil, T. El Rhafiki e Y. Mourad, Economic and environmental assessment of solar airconditioning systems in Morocco, Renewable and Sustainable Energy Reviews, 50, 770-781 (2015)

ASHRAE, HVAC, Aplications, Termal Confort (1997)

ASHRAE, Fundamentals, ASHRAE Handbook, I-P Editions (2005)

Barros, M., M. Balbis y otros cinco autores, Comparación del consumo energético entre las tecnologías de aire acondicionado tipo mini-split y volume de refrigerante variable en un edificio educativo, Rev. Espacios, 38 (43), 19 (2017)

Bom Conselho, R., R. Rodrigues, M.T. Paulino y A. Valadão, Thermal comfort of seats as visualized by infrared thermography, Applied Ergonomics, 62, 142-149 (2017)

Busch, J., Tale of two populations: thermal comfort in air-conditioned and naturally ventilated offices in Thailand, Energy and Buildings, 18(3), 235-249 (1992)

Cabello, J.J., V. Sousa y otros cuatro autores, Tools to improve forecasting and control of the electricity consumption in hotels, Journal of Cleaner Production, 137, 803-812 (2016)

Cabrera, O., A. Borroto, J. Monteagudo y C. Pérez, Evaluación del indicador kWh/HDO de eficiencia eléctrica en instalaciones hoteleras, Retos turísticos, 3(2), 38-41 (2004)

Carbonell, T., e I. Salgado, Desiccant cooling system to decrease energy consumption in Restaurant study case, 37(1), 55-62 (2016)

Djamila, H., Indoor thermal comfort predictions: Selected issues and trends, Renewable and Sustainable Energy Reviews, 74, 569-580 (2017)

Fernández, L., T. Carbonell y L. Aballe, Aplicación de Gestión Total Eficiente de Energía en el Centro Internacional de Salud "La Pradera", Ingeniería Energética, 35(2), 112-121 (2014)

Gallegos, R., N. Velázquez y A. Luna, Simulación Dinámica y Estudio Comparativo de diferentes Configuraciones de Sistemas de Enfriamiento Evaporativo para Mexicali, México, Información Tecnológica, 21(2), 45-58 (2010)

Haiguo, Y., A Li y otros tres autores, Experimental study on airflow characteristics of a square column attached ventilation mode, Building and Environment, 109, 112-120 (2016)

Klein, S.A., TRNSYS: A Transient System Simulation Program, Solar Energy Laboratory, University of Wisconsin, Madison, USA (2018)

Maroy, K., K. Carbonez, M. Steeman y N. Van Den Bossche, Assessing the thermal performance of insulating glass units with infrared thermography: Potential and limitations, Energy and Buildings, 138, 175-192 (2017)

Oldewurtel, F., A. Parisio, C. Jones, M. Morari y D. Gyalistras, Energy efficient building climate control using stochastic predictive control and weather predictions, American Control Conference (ACC), 5100-5105), Baltimore, Estados Unidos, 30 June (2010)

Privara, S., J. Cigler y otros cuatro autores, Building modeling as a crucial part for building predictive control, Energy and Buildings, 56, 8-22 (2013)

Privara, S., J. Široký, L. Ferkl y J. Cigler, Model predictive control of a building heating system: The first experience, Energy and Buildings, 43(2), 564-572 (2011)

Ramos, M. C., Determinación de estándares de confort térmico para personas que habitan en clima tropical sub-húmedo, Tesis de Magister, Universidad Internacional de Andalucía, España (2012)

Shan, K. y W. Shengwei, Energy efficient design and control of cleanroom environment control systems in subtropical regions - A comparative analysis and on-site validation, Applied Energy, 204, 582-595 (2017) 
Vakiloroaya, V., B. Samali y K. Pishghadam, Investigation of energy-efficient strategy for direct expansion air-cooled air conditioning systems, Applied Thermal Engineering, 66(1), 84-93 (2014)

Van Hoof, J., L. Schellen, V. Soebarto, J.K. Wong y J.K. Kazak, Ten questions concerning thermal comfort and ageing, Building and Environment, 120, 123-133 (2017)

Vehelts, C., Model Predictive Control of Ground Coupled Heat Pump Systems for Office Buildings. Ph.D. Dissertation, Science, Engineering and Technology Group, Department of Mechanical Engineering, Catholic University of Leuven, Belgica (2012)

Vega, B., L. Castellanos, P. Monteagudo, Modeling and identification of the cooling dynamics of a tropical island hotel, Energy and Buildings, 92, 19-28 (2015)

Viloria, A., N. Acuna, H. Mejia, M. Galofre, Determination of the Influence of Thermal Comfort in Care and Concentration of Media Education Students: Case Colombia, Indian Journal of Science and Technology, 9 (46), 1-7 (2016)

Wu, Y., S. Cisesky y otros seis autores, Fluid pressurization and tractional forces during TMJ disc loading: A biphasic finite element analysis, Orthodontics and Craniofacial Research, 20, 151-156 (2017) 
